\title{
Atorvastatin Represses the Angiotensin 2-Induced Oxidative Stress and Inflammatory Response in Dendritic Cells via the PI3K/Akt/Nrf 2 Pathway
}

\author{
Yuanji Ma, ${ }^{1,2}$ Zhaoyang Chen, ${ }^{1,2}$ Yunzeng Zou, ${ }^{1,2}$ and Junbo Ge ${ }^{1,2}$ \\ ${ }^{1}$ Shanghai Institute of Cardiovascular Diseases, Zhongshan Hospital, Fudan University, 180 Fenglin Road, Shanghai 200032, China \\ ${ }^{2}$ Institute of Biomedical Science, Fudan University, 138 Yixueyuan Road, Shanghai 200032, China \\ Correspondence should be addressed to Junbo Ge; Jbge@zs-hospital.sh.cn
}

Received 12 March 2014; Revised 11 June 2014; Accepted 11 June 2014; Published 3 July 2014

Academic Editor: Ryuichi Morishita

Copyright (C) 2014 Yuanji Ma et al. This is an open access article distributed under the Creative Commons Attribution License, which permits unrestricted use, distribution, and reproduction in any medium, provided the original work is properly cited.

Dendritic cells (DCs), which are highly proficient antigen-presenting cells, play a complex role in both the initiation and progression of atherosclerosis. We tested the hypothesis that the anti-inflammatory and antioxidant effects of atorvastatin may be partly mediated by the phosphatidylinositol 3-kinase/protein kinase B/transcription factor nuclear factor-erythroid 2-related factor 2 (PI3K/Akt/Nrf 2) pathway via the attenuation of DC maturation, thus reducing the inflammatory and oxidative stress responses. This study showed that angiotensin 2 (Ang 2) induced the maturation of DCs, stimulated CD83, CD40, CD80, and CD86 expression, and increased the secretion of IL-12p70, IL-6, and TNF- $\alpha$. These effects were suppressed by atorvastatin. Atorvastatin also lowered the levels of reactive oxygen species (ROS) and malondialdehyde (MDA), counteracting their initial increases in response to Ang 2 stimulation. Atorvastatin activated Nrf 2 via the PI3K/Akt pathway and thereby promoted Nrf 2 translocation from the cytoplasm to the nucleus in bone marrow-derived dendritic cells (BMDCs), a process that was reversed by the PI3K inhibitor LY294002. Therefore, the regulation of Nrf 2 expression by the PI3K/Akt pathway plays an important role in the regulation of the statinmediated antioxidant and anti-inflammatory responses in DCs.

\section{Introduction}

Atherosclerosis (AS) is a chronic, multifactorial disease that develops in response to inflammation and oxidative stress triggered by immune responses to autoantigens or by crossreactions to foreign antigens, triggering the formation of lesions in arterial blood vessels [1]. Dendritic cells (DCs) are antigen-presenting cells that are highly involved in the process of AS, an involvement characterized by the activation of $\mathrm{T}$ cells and the stimulation of vascular inflammatory responses $[2,3]$. The maturation of DCs is stimulated by angiotensin 2 (Ang 2), oxidized low-density lipoprotein (oxLDL), advanced glycosylation end products [3], or other antigens associated with atherosclerotic lesions [4-6], the development of which is characterized by an increasing amount of cytokine secretions and the upregulation of CD83, CD80, CD86, and CD40 [7]. Ang 2, one of the most important factors in the development of AS, mediates many cellular activities by stimulating the formation of reactive oxygen species (ROS), and it also initiates inflammatory responses by activating DCs $[4,8]$. Several studies have shown that statins exert many effects beyond lowering cholesterol to ameliorate endothelial function, such as by increasing atherosclerotic plaque stability and activating anti-inflammatory and antioxidant mediators [9-11].

Transcription factor nuclear factor-erythroid 2-related factor 2 (Nrf 2), a regulator of cellular oxidative stress [12] and inflammatory response activation [13], is a widely expressed transcription factor during AS. When exposed to ROS or other inducers, Nrf 2 is released from Kelch-like ECHassociated protein 1 (Keap 1) and translocates into the nucleus, where it induces the expression of antioxidant genes by binding to antioxidant response elements (AREs) [14], driving the transcription of many antioxidative genes and protecting cells from oxidative stress. Previous evidence showed that the phosphatidylinositol 3-kinase/protein kinase B (PI3K/Akt) 
pathway plays a role in regulating Nrf 2 activation and its subsequent nuclear translocation [15]. Moreover, the phosphorylation of Akt has been associated with the activation of Nrf 2 [16].

Our previous studies have suggested that the immune maturation of DCs may play a crucial role in atherosclerotic lesions and that DC suppression may be involved in antiatherogenic mechanisms $[6,17,18]$. Furthermore, several recent studies have shown that statins activate the PI3K/Akt pathway, resulting in the upregulation of $\operatorname{Nrf} 2[19,20]$. However, the precise biochemical and molecular mechanisms by which statins inhibit oxidative stress and inflammation are not completely understood. Therefore, the purpose of this study was to investigate the mechanisms regulating oxidative stress and inflammatory responses in DCs.

\section{Materials and Methods}

2.1. Cell Culture and Treatments. Bone marrow-derived dendritic cells (BMDCs) obtained from approximately 6-weekold C57BL/6 mice were cultured in RPMI 1640 media supplemented with $10 \mathrm{ng} / \mathrm{mL}$ granulocyte-macrophage colonystimulating factor (GM-CSF) and $1 \mathrm{ng} / \mathrm{mL}$ IL- 4 at $37^{\circ} \mathrm{C}$ in $5 \%$ humidified $\mathrm{CO}_{2}$ for $4 \mathrm{~h}$. Medium containing nonadherent cells was replaced with fresh medium every 2 days. On culture day 7, the cells were treated with either Ang 2 (100 nM) (Sigma-Aldrich, St. Louis, MO, USA) alone or in combination with various concentrations $(2.5,5$, or $10 \mu \mathrm{M})$ of atorvastatin (Sigma-Aldrich, St. Louis, MO, USA) for $24 \mathrm{~h}$. Phosphatebuffered saline (PBS) was used as a control. In the inhibitor experiment, cells were exposed to Ang 2 (100 nM) for $24 \mathrm{~h}$ after pretreatment with an inhibitor (LY294002, $100 \mathrm{nM}$ ) for $1 \mathrm{~h}$, whereas in the Nrf 2 activator experiment, cells were exposed to Ang 2 (100 nM) for $24 \mathrm{~h}$ after pretreatment with either $10 \mu \mathrm{M}$ atorvastatin or $10 \mu \mathrm{M}$ sulforaphane (SUL, Sigma-Aldrich, St. Louis, MO, USA) for $1 \mathrm{~h}$. The antibodies for Akt, phospho-Akt (Ser473), and Nrf 2 (Ser40), as well as the inhibitor LY294002, were purchased from Cell Signaling Technology (Beverly, MA, USA).

2.2. Flow Cytometric Measurement. BMDCs were washed and resuspended in ice-cold PBS containing 5\% fetal bovine serum to prevent nonspecific binding and incubated with anti-CD83, anti-CD80, anti-CD86, and anti-CD40 (BD Pharmingen, San Diego, CA, USA) for 30 minutes at $4^{\circ} \mathrm{C}$. After extensive washing, the stained cells were analyzed using a FACScan flow cytometer (BD Biosciences, San Jose, CA, USA) and Cell Quest software.

Intracellular levels of ROS were measured with DCFHDA molecular probes (Molecular Probes-Invitrogen, Carlsbad, CA, USA). Cells were incubated with $10 \mu \mathrm{M}$ DCFH$\mathrm{DA}$ for $30 \mathrm{~min}$ at $37^{\circ} \mathrm{C}$ and then washed and resuspended in PBS at $1 \times 10^{6}$ cells $/ \mathrm{mL}$. The DCs were analyzed using flow cytometry. The fluorescence was determined at 503/529 nm and expressed as a percentage of the control.

2.3. Western Immunoblotting. Protein samples were fractionated with 12\% SDS-PAGE (Invitrogen, Carlsbad, CA, USA) and transferred to polyvinylidene fluoride membranes
(Millipore, Bedford, MA, USA). The membranes with blotted protein were blocked, followed by probing with Akt, phospho-Akt (Ser473), and Nrf 2 antibodies at $4^{\circ} \mathrm{C}$ overnight. The membranes were washed and incubated at room temperature for $2 \mathrm{~h}$ with diluted (1:5000) secondary HRPconjugated antibodies. Immunoreactive proteins were identified using SuperSignal West Pico Chemiluminescent Substrate (Thermo, Franklin, MA, USA). Densitometric analysis of the western blotting was performed using Image J software. $\beta$-Actin was used as the loading control.

2.4. Enzyme-Linked Immunosorbent Assay. The supernatant of the cultured BMDCs was harvested and stored at $-70^{\circ} \mathrm{C}$. The cytokine concentrations of TNF- $\alpha$, IL-12P40, IL- 6 , and IFN- $\gamma$ were analyzed using enzyme-linked immunosorbent assay (ELISA) kits (R\&D Systems, Minneapolis, MN, USA) according to the manufacturer's instructions. The superoxide dismutase (SOD) activity and MDA contents were measured at 450 and $532 \mathrm{~nm}$ by SOD and MDA ELISA kits (R\&D Systems, Minneapolis, MN, USA), respectively.

2.5. T Cell Proliferation Assays. The effects of atorvastatin on $\mathrm{T}$ cell proliferation mediated by Ang 2-treated BMDCs were analyzed in a mixed lymphocyte reaction. Adult $\mathrm{T}$ cells from the spleens of C57BL/6 mice were purified using a Dynal Mouse T Cell Negative Isolation Kit (Invitrogen, Paisley, UK). BMDCs were added to $1 \times 10^{5} \mathrm{~T}$ cells at a ratio of 1:10 (DCs:T cells) and incubated for 5 days in 96-well tissue culture plates. The cells were incubated with $20 \mu \mathrm{L}$ of diluted bromodeoxyuridine (BrdU) (Chemicon, Temecula, CA, USA) for $18 \mathrm{~h}$ and were then analyzed on a spectrophotometer microplate reader at $490 \mathrm{~nm}$, according to the manufacturer's instructions.

2.6. Statistical Analyses. The data are presented as the means \pm SD, with $P<0.05$ considered to be statistically significant. A one-way ANOVA, followed by the Student-Newman-Keuls test, was employed for the statistical analysis of our results. All statistical analyses were performed with SPSS 11.5 statistical software.

\section{Results}

3.1. Atorvastatin Attenuates Ang 2-Induced Oxidative Stress in BMDCs. To investigate how atorvastatin affects Ang 2induced oxidative stress in BMDCs, we used flow cytometry to measure the intracellular level of ROS in response to Ang 2 stimulation, and we also measured the levels of MDA and SOD with ELISA kits. As depicted in Figure 1, $100 \mathrm{nM}$ Ang 2 exhibited a stimulating effect on ROS (a) and MDA production (c) but inhibited SOD activity. Cotreatment with atorvastatin with concentrations ranging from 2.5 to $10 \mu \mathrm{M}$ reduced intracellular ROS production in a dose-dependent manner, and similar results were observed with MDA. However, atorvastatin suppressed the Ang 2-induced inhibition of SOD activity (b). Therefore, these results suggest that atorvastatin significantly suppresses Ang 2-induced oxidative stress in BMDCs. 


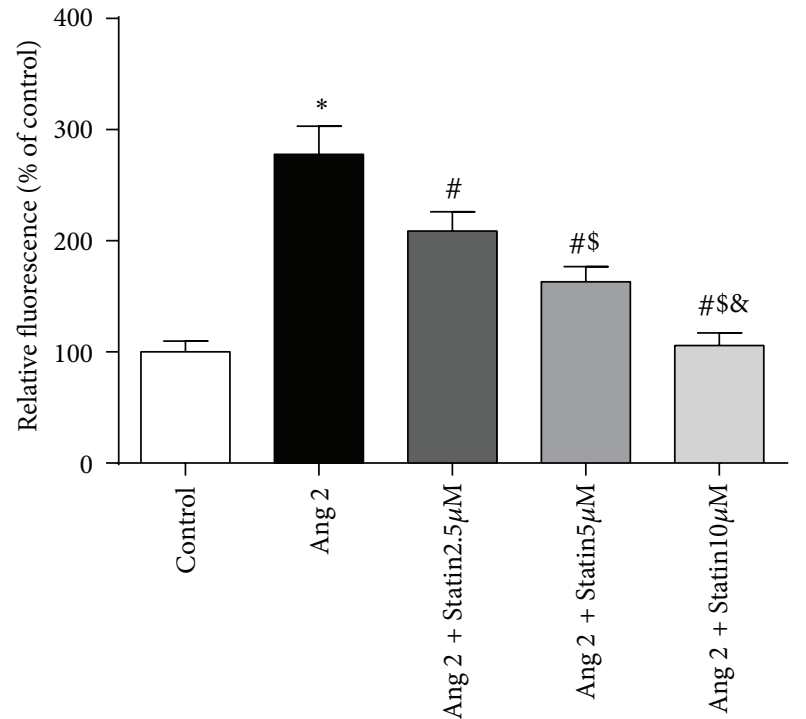

(a)

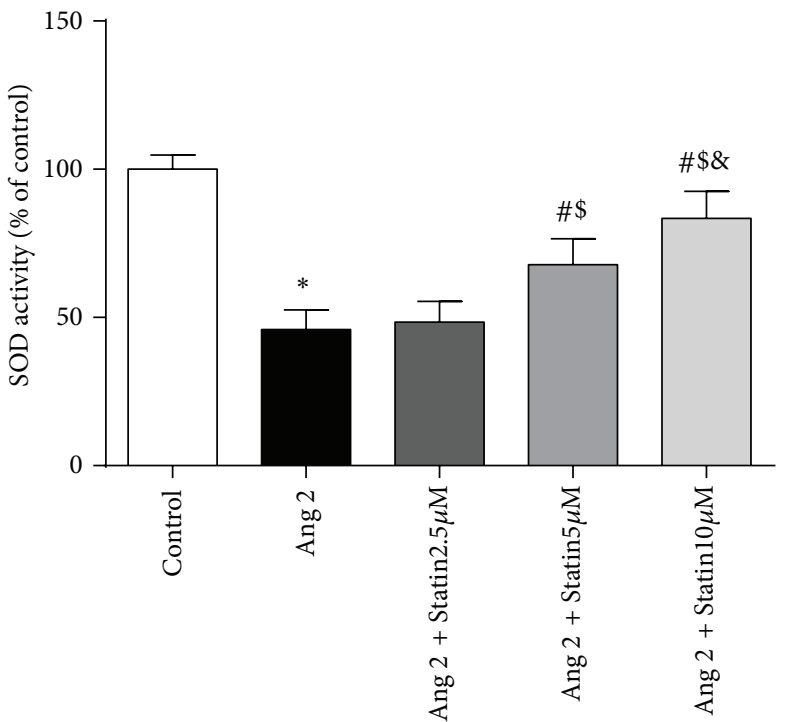

(b)

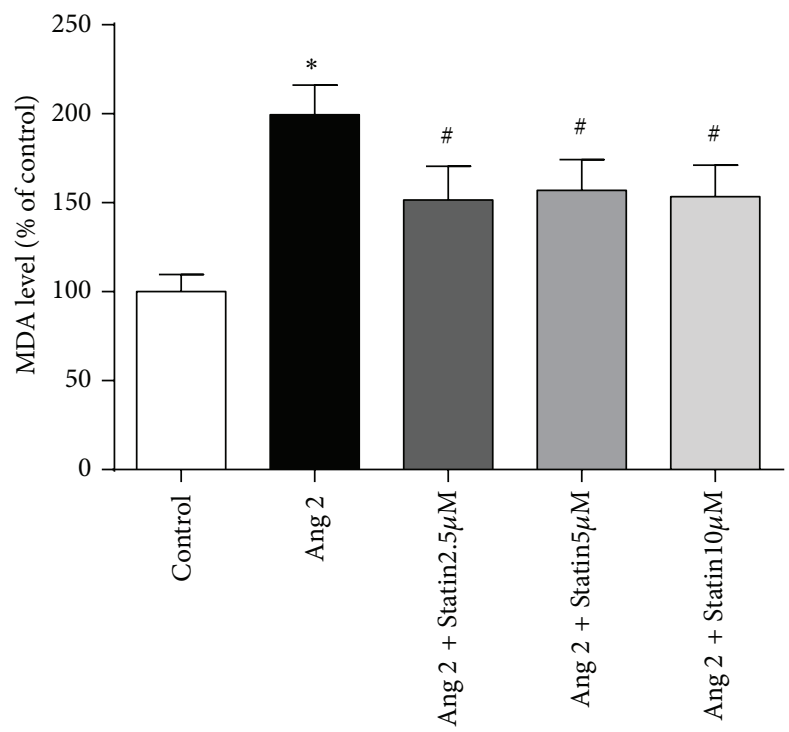

(c)

FIGURE 1: Effects of atorvastatin on ROS formation (a), SOD activity (b), and MDA levels (c) in BMDCs in the presence of Ang 2. BMDCs were incubated with Ang $2(100 \mathrm{nM})$ alone or in combination with various concentrations $(2.5,5$, or $10 \mu \mathrm{M})$ of atorvastatin for $24 \mathrm{~h}$. (a) The intracellular ROS levels were measured via flow cytometry using DCFH-DA. (b) The SOD activity and (c) MDA levels were measured at 450 and $532 \mathrm{~nm}$, respectively, using ELISA kits. The data are shown as the mean $\pm(\mathrm{SD})(n=3) ;{ }^{*} P<0.05$ versus control; ${ }^{\#} P<0.05$ versus Ang 2 group; ${ }^{\$} P<0.05$ versus Ang $2+2.5 \mu \mathrm{M}$ atorvastatin group; ${ }^{\circledR} P<0.05$ versus Ang $2+5 \mu \mathrm{M}$ atorvastatin group.

\subsection{Effect of Atorvastatin on Ang 2-Induced BMDC Matura-} tion and Inflammatory Cytokine Secretion. There are many costimulatory proteins on the surface of BMDCs, including CD40, CD83, CD80, and CD86. Therefore, these proteins were examined in Ang 2-treated $(100 \mathrm{nM})$ BMDCs that were exposed to various concentrations of atorvastatin (2.5, 5 , or $10 \mu \mathrm{M})$. The flow cytometry results showed that the expression of the cell-surface markers CD40, CD83, CD80, and CD86 was upregulated by Ang 2. We subsequently observed that atorvastatin significantly downregulates CD83 and CD40 expression but exerts moderate inhibitory effects on CD86 and CD80. These results indicate that atorvastatin may suppress Ang 2-induced DC maturation (Figure 2(a)).
An analysis of cytokine levels, that is, TNF- $\alpha$, IL-12P40, IL-6, and IFN- $\gamma$, indicated an Ang 2-induced inflammatory response in BMDCs. We found that the secretion of TNF- $\alpha$, IL-6, and IL-12p70 was increased significantly in the presence of Ang 2. In addition, the secretion of these cytokines was suppressed by atorvastatin in a dose-dependent manner. However, IFN- $\gamma$ was clearly not affected by either Ang 2 or atorvastatin (Figure 2(b)).

3.3. Atorvastatin Activates Nrf 2 through the PI3K/Akt Pathway in BMDCs. Recent studies have demonstrated that statins activate the PI3K/Akt pathway $[19,20]$. To confirm these findings, we monitored the phosphorylation of Akt at 


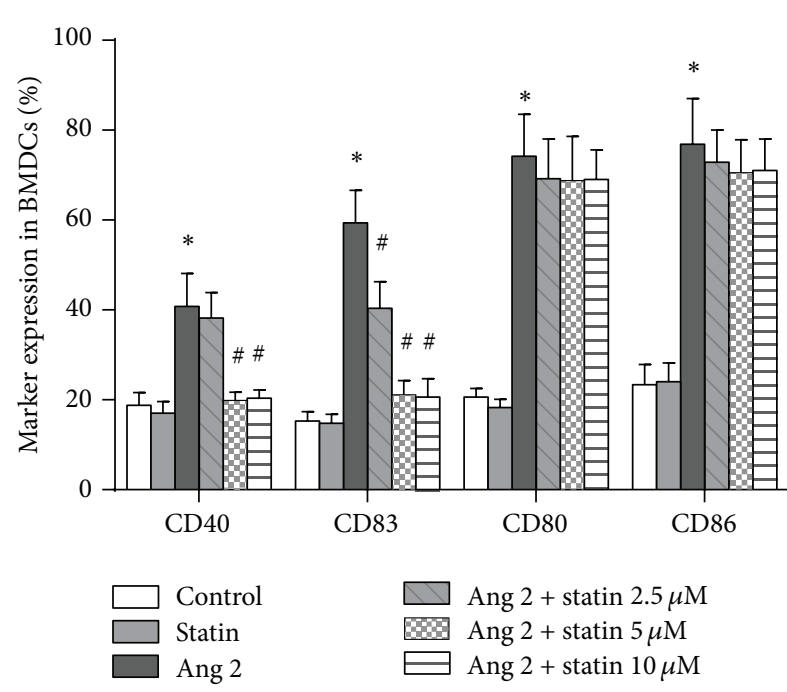

(a)

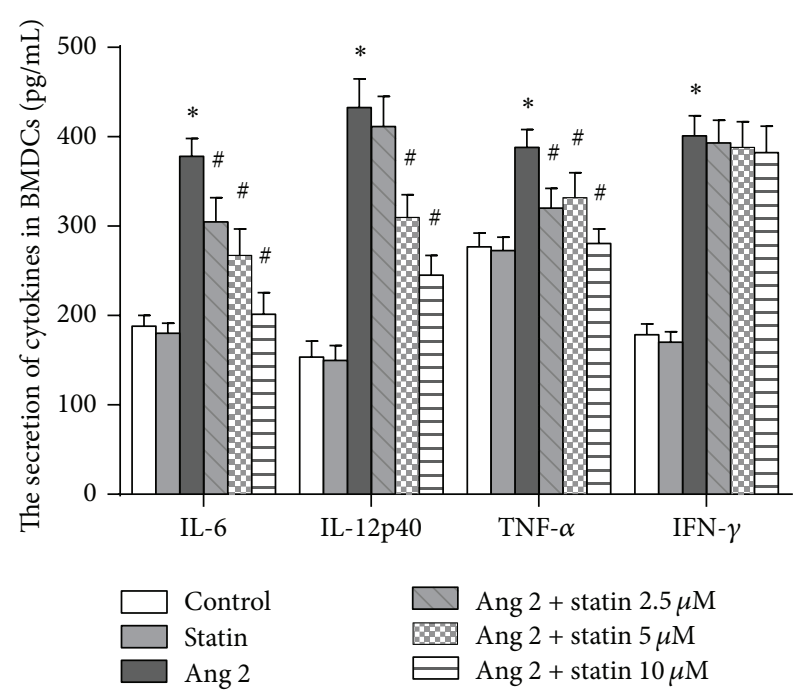

(b)

FIGURE 2: Inhibitory effects of atorvastatin on Ang 2-induced BMDC maturation and inflammatory responses. After pretreatment with either PBS or Ang $2(100 \mathrm{nM})$, BMDCs were exposed to various concentrations of atorvastatin $(2.5,5$, or $10 \mu \mathrm{M})$ for $24 \mathrm{~h}$. (a) Expression of the cellsurface markers CD40, CD83, CD80, and CD86, as determined by flow cytometry $(n=3)$. (b) Expression of cytokines in BMDCs analyzed by ELISA $(n=3)$. The data are shown as the mean $\pm(\mathrm{SD})(n=3) ;{ }^{*} P<0.05$ versus control; ${ }^{*} P<0.05$ versus Ang 2 group.

position Ser473 by western blotting in atorvastatin-treated BMDCs. Surprisingly, as shown in Figure 3(a), we found that atorvastatin promoted Akt phosphorylation in a timedependent manner.

Nrf 2 plays a central role in the cellular antioxidative system. The nuclear accumulation of Nrf 2 is considered to be a marker of Nrf 2 activation in response to stressors. Moreover, the PI3K/Akt pathway is involved in regulating Nrf 2 activation and the subsequent nuclear translocation of Nrf 2 [15]. Therefore, we further investigated the molecular mechanisms underlying the effects of atorvastatin on Nrf 2 activation. To address this question, BMDCs were treated with $100 \mathrm{nM}$ Ang 2 in the presence or absence of $10 \mu \mathrm{M}$ atorvastatin, and the cell lysate was fractionated into cytoplasmic and nuclear fractions. The fractions were then analyzed by western blotting for Nrf 2. Western blotting (Figure 3(b)) revealed that the level of Nrf 2 in the cytoplasmic fraction was significantly reduced, compared with the control, by both atorvastatin alone and atorvastatin in combination with Ang 2. By contrast, the level of Nrf 2 in the nuclear fraction was increased under the same conditions. These results demonstrate that atorvastatin promoted the nuclear translocation of Nrf 2 in Ang 2-treated BMDCs, by increasing both the dissociation of Nrf 2 from Keap 1 and the translocation of Nrf 2 to the nucleus [14].

Next, we used LY294002 (100 nM), a PI3K inhibitor, to assess whether this pathway is involved in Nrf 2 activation by atorvastatin. Western blot analysis showed that, in Ang 2treated BMDCs, atorvastatin promoted the phosphorylation of Akt and the translocation of Nrf 2. Treatment of the cells with the PI3K inhibitor LY294002 blocked these effects.

To further confirm the involvement of Nrf 2 activation in atorvastatin-mediated actions against Ang 2-induced oxidative stress, we used the Nrf 2 activator SUL to evaluate whether atorvastatin has an effect on the translocation of Nrf 2 into the nucleus in BMDCs. As shown in Figure 3(c), western blotting analysis confirmed that similar to SUL, which is a known $\operatorname{Nrf} 2$ activator, atorvastatin induced the translocation of Nrf 2 into the nucleus. However, while atorvastatin promoted Akt phosphorylation, SUL did not. These results, which are shown in Figure 3, indicate that the PI3K/Akt/Nrf 2 pathway is involved in the atorvastatinmediated inhibition of Ang 2-induced cellular responses.

\subsection{PI3K/Akt Pathway Involvement in Ang 2-Induced Oxida-} tive Stress and Inflammatory Responses Is Suppressed by Atorvastatin. Because atorvastatin activates the PI3K/Akt/Nrf 2 pathway, we investigated whether atorvastatin influences the course of Ang 2-induced oxidative stress and inflammatory responses. We found that atorvastatin and SUL had inhibitory effects on ROS and MDA production but stimulated SOD activity. In addition, the PI3K inhibitor LY294002 significantly diminished the antioxidant effects of atorvastatin (Figures 4(a), 4(b), and 4(c)).

We next studied the atorvastatin-mediated inhibition of Ang 2-induced inflammation. We found that SUL had the same effect on DC maturation and inflammatory cytokines as atorvastatin. However, the pretreatment of cells with $100 \mathrm{nM}$ LY294002 abrogated the influence of atorvastatin on DC maturation and inflammatory cytokine mediators, such as CD83, CD40, TNF- $\alpha$, IL-6, and IL-12p70 (Figures 4(d) and 4(e)). These results indicate the involvement of the PI3K/Akt/Nrf 2 pathway in BMDC maturation and inflammation.

\subsection{Atorvastatin Attenuated the Ability of Ang 2-Treated} BMDCs to Induce T Cell Proliferation. To further demonstrate the physiological relevance of our findings, we evaluated the effect of atorvastatin on antigen presentation. As 


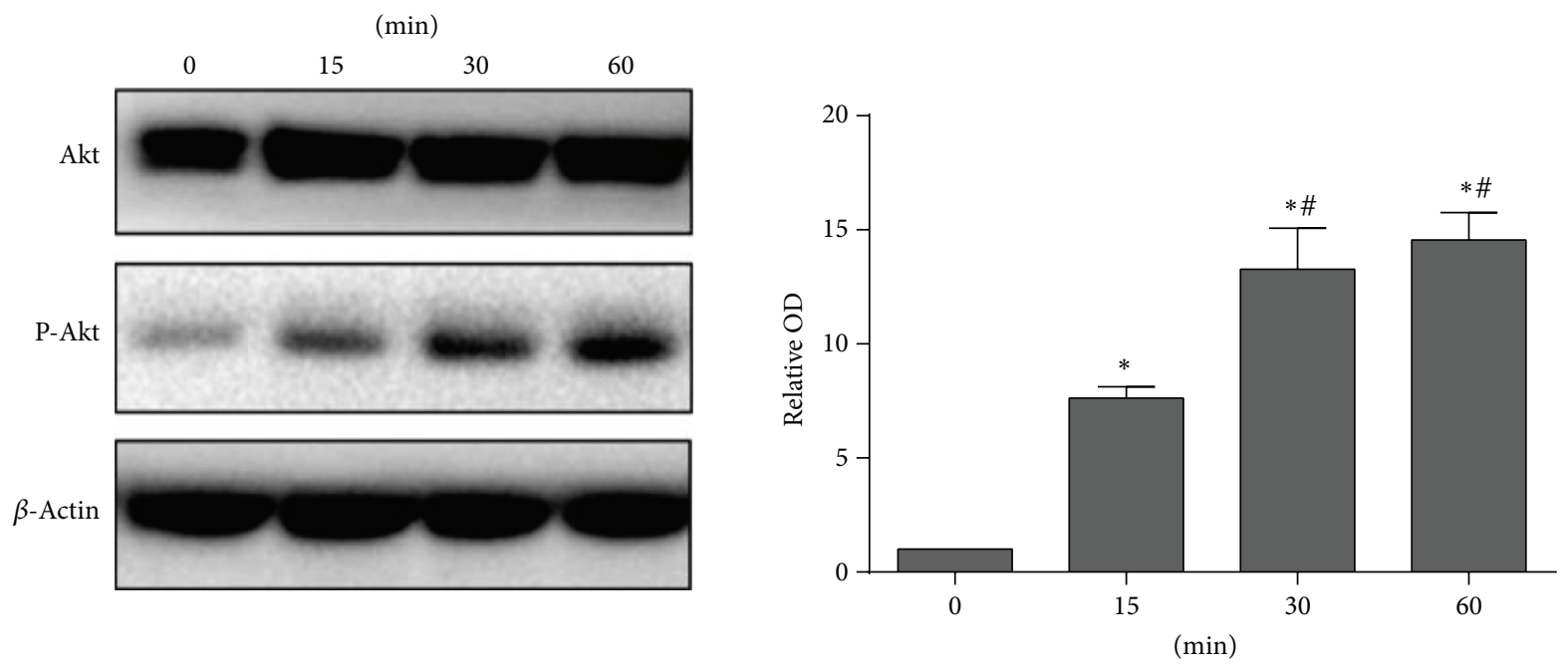

(a)
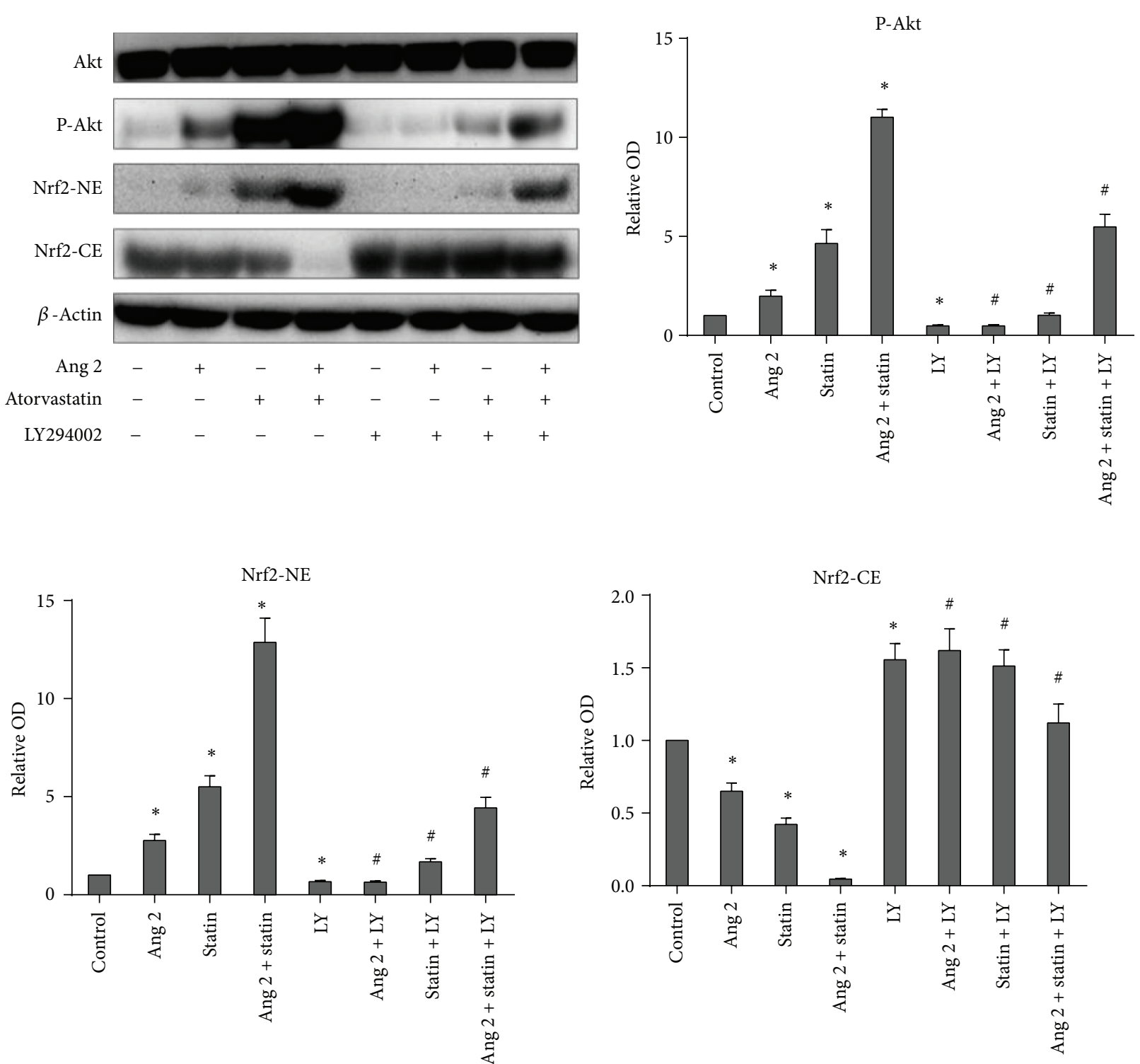

(b)

FIgure 3: Continued. 

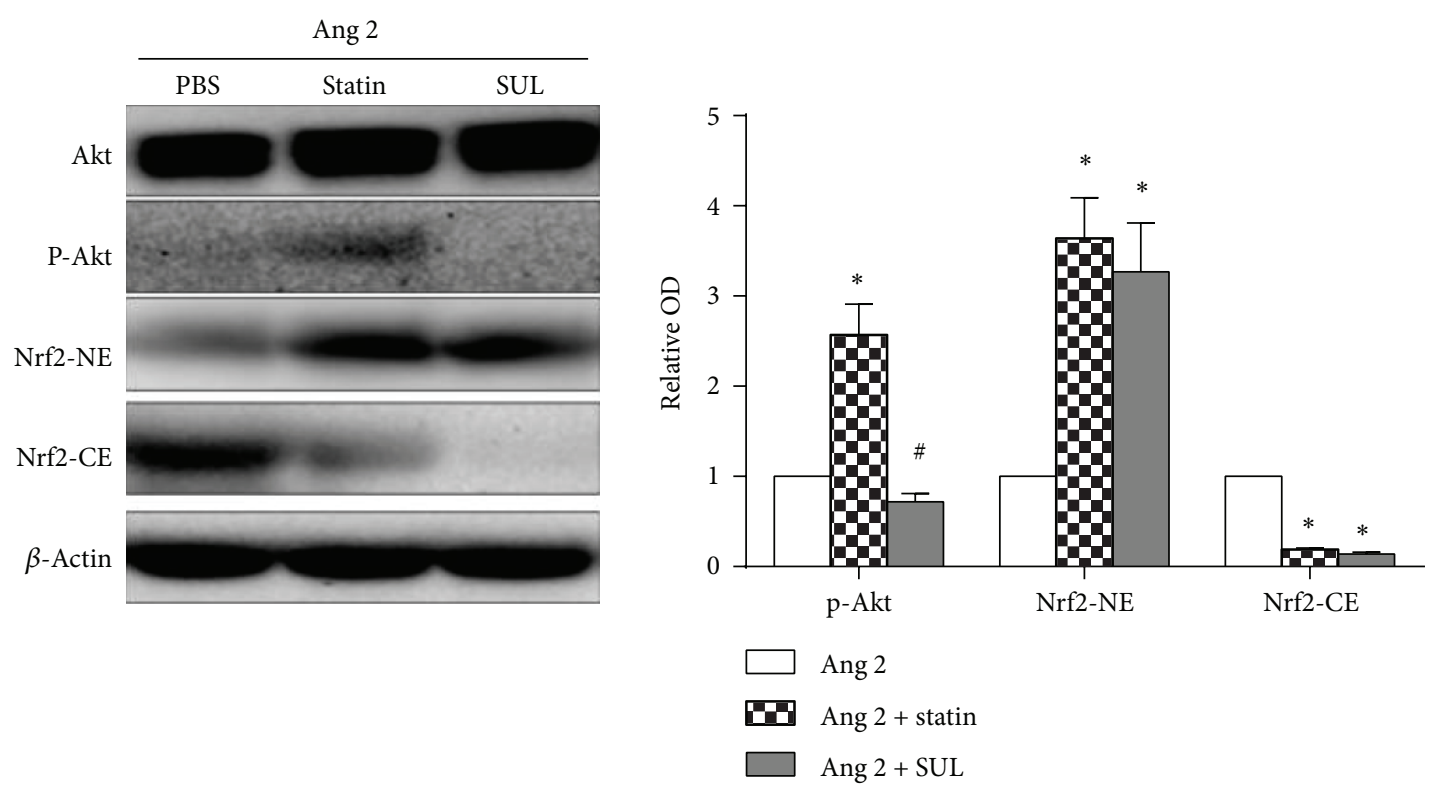

(c)

Figure 3: PI3K/Akt/Nrf 2 pathway activation mediates the protective effect of atorvastatin in BMDCs. (a) Atorvastatin promotes Akt phosphorylation in a time-dependent manner. BMDCs were treated with $10 \mu \mathrm{M}$ atorvastatin for the indicated times. $\beta$-Actin was employed as a loading control. The graph represents the means $\pm(\mathrm{SD})(n=3),{ }^{*} P<0.05$ versus time $0 \mathrm{~min} ;{ }^{\#} P<0.05$ versus time 15 min. (b) The nuclear translocation of Nrf 2 was mediated by PI3K/Akt signals in atorvastatin-treated cells. BMDCs were treated with $100 \mathrm{nM}$ Ang 2 in the presence or absence of $10 \mu \mathrm{M}$ atorvastatin. Cellular cytoplasmic extracts (CE) and nuclear extracts (NE) were separated on a 12\% SDSPAGE gel to probe Nrf 2 and actin levels by western blotting. Total extracts of the cells were prepared, and the p-Akt (Ser473) expression was assayed by western immunoblotting. In the presence of $100 \mathrm{nM} \mathrm{LY294002,} \mathrm{the} \mathrm{effects} \mathrm{of} \mathrm{atorvastatin} \mathrm{on} \mathrm{both} \mathrm{Akt} \mathrm{phosphorylation} \mathrm{and} \mathrm{Nrf}$ 2 translocation were inhibited. (c) Nrf 2 activation was involved in the atorvastatin-mediated inhibition of Ang 2-induced oxidative stress. Atorvastatin induced the translocation of Nrf 2 into the nucleus and the phosphorylation of Akt. However, with $10 \mu \mathrm{M}$ SUL, we observed only Nrf 2 activation and not Akt phosphorylation. $\beta$-Actin was employed as a loading control. The data represent the means \pm SD $(n=3)$ from three independent repeats; ${ }^{*} P<0.05$ versus control; ${ }^{*} P<0.05$ versus Ang $2+$ atorvastatin group.

shown in Figure 4(f), $\mathrm{T}$ cell proliferation was detected by BrdU, and BMDCs exposed to Ang 2 effectively induced allogeneic T cell proliferation compared with controls. However, $10 \mu \mathrm{M}$ atorvastatin almost completely blocked Ang 2-induced T cell activation.

\section{Discussion}

The major finding of our study is that atorvastatin represses the oxidative stress induced by Ang 2 in BMDCs via the PI3K/Akt/Nrf 2 pathway. Atorvastatin also inhibited Ang 2induced inflammatory responses via the downregulation of BMDC surface markers and inflammatory cytokines. Additionally, blocking the PI3K/Akt/Nrf 2 pathway may abrogate the effects of atorvastatin on BMDCs during inflammation and oxidative stress.

Previous studies by our group indicated that mature DCs may play key roles in the initiation and regulation of immune responses involved in atherogenesis, as evidenced by the antiatherogenic effects of DC suppression $[6,17,18]$. Statins, the cornerstone of antiatherogenic therapy, have an effect on DC maturation [21], but the mechanisms underlying this effect remain unclear. In this study, we focused on the impact of Ang 2 on DC activation. In doing so, we investigated the influence of atorvastatin on DC maturation and cytokine production induced by Ang 2. CD83, CD40, and CD80/CD86 are characteristic surface markers in mature DCs. CD83 is preferentially expressed on mature DCs [22], whereas CD40 is a member of the TNF receptor family, which is transiently expressed on T cells under inflammatory conditions [23]. We found that atorvastatin downregulates the expression of both CD83 and CD40, the expression of which is stimulated by Ang 2. This indicates that atorvastatin attenuates atherosclerotic lesions by downregulating DC surface molecules. Additionally, atorvastatin appears to inhibit DC maturation and antigen uptake and presentation, which are induced by LPS, Ang 2 , and proinflammatory factors, and facilitate an antigenspecific $\mathrm{T}$ cell immune response $[24,25]$. In addition, we found that statins suppress Ang 2-induced allogeneic T cell proliferation. Finally, the inflammatory activation of DCs is also reflected by the differential expression of the cytokines induced by Ang 2. We discovered that Ang 2 increased the expression of the proinflammatory cytokines IL-12p70, IL6 , and TNF- $\alpha$. By contrast, atorvastatin inhibited this Ang 2 -induced increase. Surprisingly, all of these effects on both DC maturation and inflammation were reversed following the addition of the PI3K inhibitor, LY294002. Therefore, the $\mathrm{PI} 3 \mathrm{~K} / \mathrm{Akt}$ pathway is involved in the atorvastatin-mediated suppression of DC maturation and inflammation. 


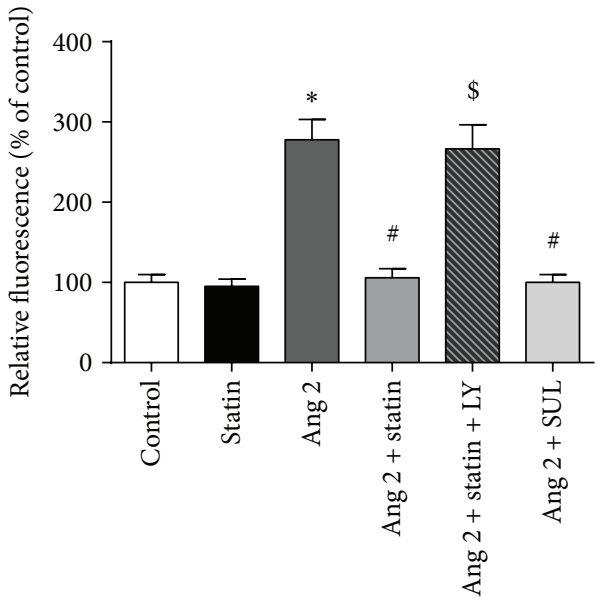

(a)

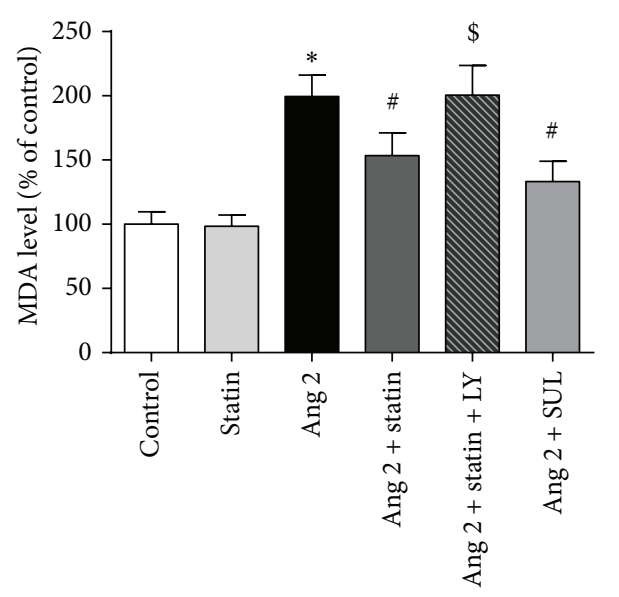

(c)

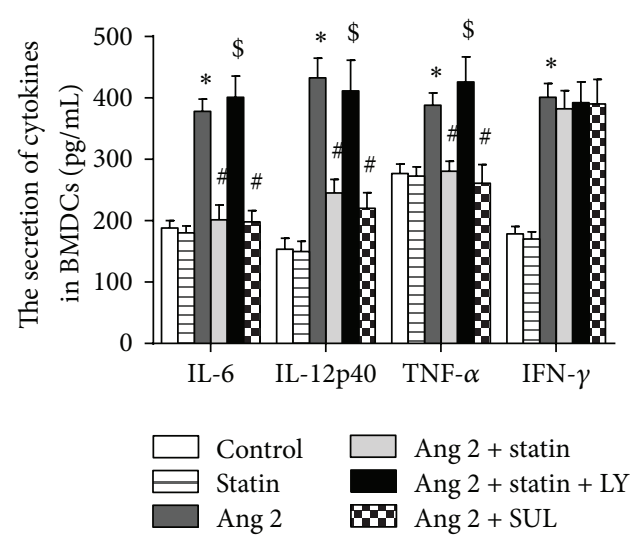

(e)

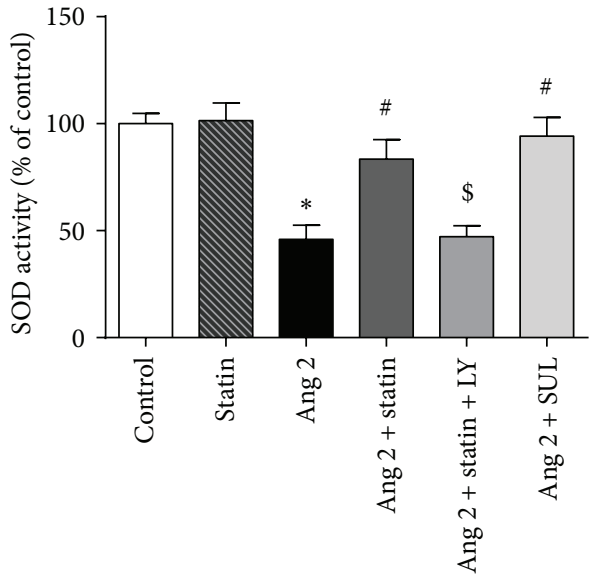

(b)
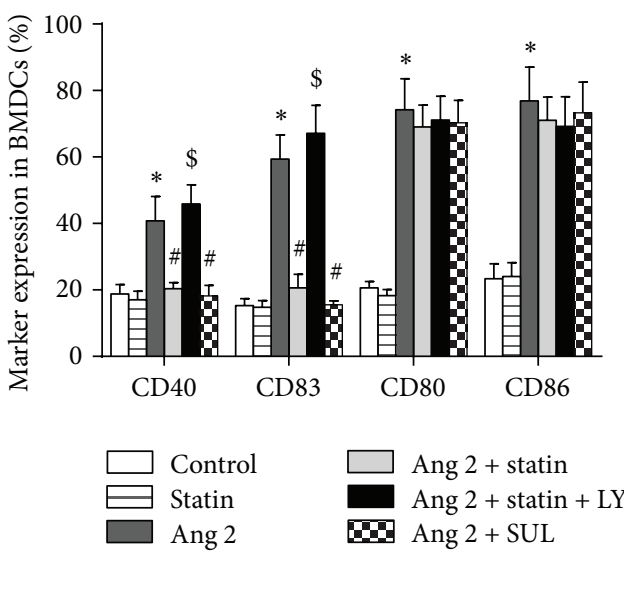

(d)
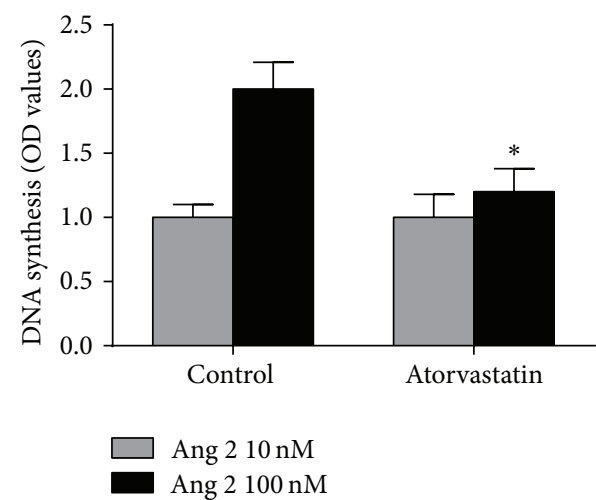

(f)

Figure 4: The PI3K/Akt/Nrf 2 pathway is involved in the atorvastatin-mediated inhibition of Ang 2-induced cellular responses. After pretreatment with PBS and either the Nrf 2 activator SUL $(10 \mu \mathrm{M})$ or the PI3K inhibitor LY294002 (100 nM) for 1h, BMDCs were incubated for $24 \mathrm{~h}$ with Ang $2(100 \mathrm{nM})$ alone, atorvastatin $(10 \mu \mathrm{M})$ alone, or Ang $2(100 \mathrm{nM})$ and atorvastatin $(10 \mu \mathrm{M})$ combined. We observed markers of oxidative stress and inflammation in the BMDCs. (a) Intracellular ROS levels were measured with flow cytometry using DCFH-DA. (b) SOD activity and (c) MDA levels were measured by ELISA kits. (d) Expression of the cell-surface markers CD40, CD83, CD80, and CD86 as determined by flow cytometry $(n=3)$. (e) Expression of the cytokines in BMDCs analyzed by ELISA $(n=3)$. (f) Atorvastatin decreased the ability of BMDCs to activate T cells. BMDCs were cultured with $100 \mathrm{nM}$ Ang 2 for $48 \mathrm{~h}$ in the absence or presence of $10 \mu \mathrm{M}$ atorvastatin. Cells were harvested and then incubated with $1 \times 10^{5} \mathrm{~T}$ cells in a 96-well plate at a ratio of $1: 10$ (BMDCs: T cells) for 5 days. For the final $18 \mathrm{~h}$ of incubation, $20 \mu \mathrm{L}$ of diluted BrdU was added to the appropriate wells $(n=3)$. The data are shown as the means $\pm(\mathrm{SD})(n=3)$; ${ }^{*} P<0.05$ versus control; ${ }^{\#} P<0.05$ versus Ang 2 group; ${ }^{\$} P<0.05$ versus Ang $2+$ atorvastatin group. 
SUL activity is explained primarily by its activation of the Nrf 2-ARE pathway [26]. In this study, the administration of SUL resulted in a significant increase in the translocation of Nrf 2 to the nucleus without activating Akt. After SUL administration, the release of the inflammatory cytokines IL12 p70, IL- 6 , and TNF- $\alpha$ and the expression of the surface markers CD83 and CD40 were attenuated. The effect of SUL was reflected further by the augmentation of SOD activity with an accompanying decrease in both the MDA and ROS levels. SUL enhanced the activity of the Nrf 2-ARE pathway, suppressing the inflammatory responses and oxidative stress induced by Ang 2.

Oxidative stress resulting from uncontrolled ROS production has been implicated in the pathogenesis of atherosclerosis. Several studies have explained the potential beneficial pleiotropic and antioxidant effects of statins [9-11]. The PI3K/Akt/Nrf 2 pathway has been shown to be involved in the antioxidant effects of statins $[19,20]$. However, the effects and mechanisms by which statins interact with DCs are less well studied, particularly where antioxidants are concerned. In this study, we showed that atorvastatin lowers ROS and MDA levels via regulation of the PI3K/Akt/Nrf 2 pathway.

To mitigate the cumulative burden of oxidative stress, cells generally utilize antioxidant defense systems and scavenge ROS and MDA. SOD is an important antioxidant enzyme. Our study indicated that DCs treated with Ang 2 showed a marked increase in oxidative stress, as evidenced by excessive ROS and MDA production. However, cotreatment with atorvastatin significantly attenuated oxidative damage induced by Ang 2, as reflected by the augmentation of SOD activity and the accompanying decrease in MDA and ROS levels. There are a few mechanisms by which Ang 2 could downregulate SOD activity. The inhibition of small G proteins, such as the NADPH oxidase component, Racl [27, 28 ], and the reduced expression of NADPH oxidase subunits [28], may mediate the antioxidant effects of atorvastatin. Ang 2 triggers NADH/NADPH-oxidase and local vascular $\mathrm{O}_{2}{ }^{--}$production [29-31]. It was recently demonstrated that Ang 2 increases Mn-SOD, diminishes CuZn-SOD, and does not alter EC-SOD expression [32]. In our study, atorvastatin treatment reversed the alterations observed during oxidative stress, a reversal most likely due to an increase in antioxidant defenses and diminished NADPH oxidase expression. In addition, we observed increased SOD activity with atorvastatin treatment.

Additionally, Nrf 2 is a regulator of cellular oxidative stress and inflammatory activity. It is sequestered in the cytoplasm as an inactive complex with its cytosolic repressor, Keap 1. The dissociation of Nrf 2 from Keap 1 is a prerequisite for the nuclear translocation and subsequent DNA binding of Nrf 2 [14]. The dissociation of the Nrf 2-Keap 1 complex, which is mediated via one or more upstream kinases, including MAPKs, PI3K/Akt, and PKC, was recently reviewed $[15,16,33]$. We confirmed that atorvastatin activates the PI3K/Akt pathway, further showing that this pathway is a prerequisite for the activation of $\mathrm{Nrf} 2$. Atorvastatin promotes both Akt phosphorylation and Nrf 2 translocation in Ang 2-treated DCs. We also observed Nrf 2 translocation, but not Akt phosphorylation, after replacing atorvastatin with the Nrf 2 activator SUL. Moreover, the PI3K inhibitor LY294002 significantly repressed the stimulatory effects of atorvastatin on $\mathrm{Nrf} 2$ activation. Therefore, these results show that the PI3K/Akt pathway partially regulates $\mathrm{Nrf} 2$ translocation, which is consistent with a prior finding in $\mathrm{H} 9 \mathrm{c} 2$ cardiomyocytes [34], thereby regulating oxidative stress and inflammation. It seems probable that atorvastatin activates the PI3K/Akt pathway via Akt phosphorylation at position Ser473, mediating Nrf 2 activation.

Blocking the PI3K/Akt/Nrf 2 pathway may repress not only the inflammatory reactions but also the oxidative stress induced by Ang 2. Oxidative signals play crucial roles in the pathogenesis of chronic inflammatory diseases by mediating the expression of inflammatory genes, such as MCP-1 and VCAM-1. Previous studies have demonstrated that the expression of Nrf 2 suppresses TNF- $\alpha$-induced MCP-1 and VCAM-1 expression, monocyte adhesion to endothelial cells, and the activation of p38 MAPK [35]. Nrf 2 also regulates a set of antioxidant proteins, such as heme oxygenase-1 (HO-1), that includes important factors for modulating inflammatory responses [36]. The overexpression of HO-1 has been shown to suppress oxidized LDL-induced monocyte transmigration, inhibiting atherosclerotic lesion formation in LDL-receptor knockout mice $[37,38]$. The coordinated regulation of these genes has synergistic effects on the maintenance of intracellular antioxidant capacity and the inhibition of inflammatory responses. However, further research of the mechanisms mediating inflammation and oxidative stress is still needed.

In summary, our results demonstrate that atorvastatin inhibits the oxidative stress and inflammatory responses induced by Ang 2 via the PI3K/Akt/Nrf 2 pathway. Furthermore, the activation of Nrf 2 by atorvastatin may be a means of both antioxidant protection and the suppression of redoxsensitive inflammatory genes, suggesting that targeting Nrf 2 activation may represent a novel therapeutic approach in the treatment of atherosclerosis.

\section{Abbreviations}

$\begin{array}{ll}\text { DC: } & \text { Dendritic cell } \\ \text { AS: } & \text { Atherosclerosis } \\ \text { Ang 2: } & \text { Angiotensin } 2 \\ \text { ROS: } & \text { Reactive oxygen species } \\ \text { MDA: } & \text { Malondialdehyde } \\ \text { oxLDL: } & \text { Oxidized low-density lipoprotein } \\ \text { Nrf 2: } & \text { Transcription factor nuclear } \\ & \text { factor-erythroid 2-related factor } 2 \\ \text { Keap 1: } & \text { Kelch-like ECH-associated protein } 1 \\ \text { ARE: } & \text { Antioxidant response element } \\ \text { PI3K: } & \text { Phosphatidylinositol 3-kinase } \\ \text { Akt: } & \text { Protein kinase B } \\ \text { BMDCs: } & \text { Bone marrow-derived dendritic cells } \\ \text { GM-CSF: } & \text { Granulocyte-macrophage } \\ & \text { colony-stimulating factor } \\ \text { SUL: } & \text { Sulforaphane } \\ \text { PBS: } & \text { Phosphate-buffered saline } \\ \text { IL: } & \text { Interleukin } \\ \text { ELISA: } & \text { Enzyme-linked immunosorbent assay } \\ \text { BrdU: } & \text { Bromodeoxyuridine }\end{array}$


SOD: Superoxide dismutase

TNF- $\alpha$ : Tumor necrosis factor- $\alpha$

IFN- $\gamma$ : Interferon $\gamma$

CE: Cytoplasmic extracts

NE: Nuclear extracts

HO: Heme oxygenase

VCAM: Vascular cell adhesion molecule-1

MCP: Monocyte chemotactic protein

MAPK: Mitogen-activated protein kinase.

\section{Conflict of Interests}

The authors declare that there is no conflict of interests in relation to the present paper.

\section{Authors' Contribution}

Yuanji Ma and Zhaoyang Chen contributed equally to this paper.

\section{Acknowledgments}

This project was supported by the General Program (Key Program, Major Research Plan) of the National Natural Science Foundation of China, Grant 81230007. The authors also thank Nature Publishing Group for English language editing of the paper.

\section{References}

[1] G. K. Hansson, "Inflammation, atherosclerosis, and coronary artery disease," The New England Journal of Medicine, vol. 352, no. 16, pp. 1685-1695, 2005.

[2] W. Cao, Y. V. Bobryshev, R. S. Lord, R. E. Oakley, S. H. Lee, and J. $\mathrm{Lu}$, "Dendritic cells in the arterial wall expresses Clq: potential significance in atherogenesis," Cardiovascular Research, vol. 60, no. 1, pp. 175-186, 2003.

[3] Y. V. Bobryshev, "Dendritic cells in atherosclerosis: current status of the problem and clinical relevance," European Heart Journal, vol. 26, no. 17, pp. 1700-1704, 2005.

[4] K. A. Nahmod, M. E. Vermeulen, S. Raiden et al., "Control of dendritic cell differentiation by angiotensin II," The FASEB Journal, vol. 17, no. 3, pp. 491-493, 2003.

[5] J. Sun, K. Hartvigsen, M. Y. Chou et al., "Deficiency of antigenpresenting cell invariant chain reduces atherosclerosis in mice," Circulation, vol. 122, no. 8, pp. 808-820, 2010.

[6] J. Ge, Q. Jia, C. Liang et al., "Advanced glycosylation end products might promote atherosclerosis through inducing the immune maturation of dendritic cells," Arteriosclerosis, Thrombosis, and Vascular Biology, vol. 25, no. 10, pp. 2157-2163, 2005.

[7] RR. Packard, E. Maganto-García, I. Gotsman et al., "CD11c(+) dendritic cells maintain antigen processing, presentation capabilities, and CD4(+) T-cell priming efficacy under hypercholesterolemic conditions associated with atherosclerosis," Circulation Research, vol. 103, no. 9, pp. 965-973, 2008.

[8] E. L. Schiffrin and R. M. Touyz, "From bedside to bench to bedside: role of renin-angiotensin-aldosterone system in remodeling of resistance arteries in hypertension," The American Journal of Physiology: Heart and Circulatory Physiology, vol. 287, no. 2, pp. H435-H446, 2004.
[9] P. Sicard, S. Delemasure, C. Korandji et al., "Anti-hypertensive effects of rosuvastatin are associated with decreased inflammation and oxidative stress markers in hypertensive rats," Free Radical Research, vol. 42, no. 3, pp. 226-236, 2008.

[10] O. Adam and U. Laufs, "Antioxidative effects of statins," Archives of Toxicology, vol. 82, no. 12, pp. 885-892, 2008.

[11] M.-S. Zhou, E. A. Jaimes, and L. Raij, "Atorvastatin prevents end-organ injury in salt-sensitive hypertension: Role of eNOS and oxidant stress," Hypertension, vol. 44, no. 2, pp. 186-190, 2004.

[12] A. R. Collins, C. J. Lyon, X. Xia et al., "Age-accelerated atherosclerosis correlates with failure to upregulate antioxidant genes," Circulation Research, vol. 104, no. 6, pp. e42-e54, 2009.

[13] S. Freigang, F. Ampenberger, G. Spohn et al., "Nrf2 is essential for cholesterol crystal-induced inflammasome activation and exacerbation of atherosclerosis," European Journal of Immunology, vol. 41, no. 7, pp. 2040-2051, 2011.

[14] T. Ishii, K. Itoh, S. Takahashi et al., "Transcription factor Nrf2 coordinately regulates a group of oxidative stress-inducible genes in macrophages," The Journal of Biological Chemistry, vol. 275, no. 21, pp. 16023-16029, 2000.

[15] Y. P. Hwang and H. G. Jeong, "Ginsenoside Rb1 protects against 6-hydroxydopamine-induced oxidative stress by increasing heme oxygenase-1 expression through an estrogen receptor-related PI3K/Akt/Nrf2-dependent pathway in human dopaminergic cells," Toxicology and Applied Pharmacology, vol. 242, no. 1, pp. 18-28, 2010.

[16] C. N. Nguyen, H. E. Kim, and S. G. Lee, "Caffeoylserotonin protects human keratinocyte $\mathrm{HaCaT}$ cells against $\mathrm{H}_{2} \mathrm{O}_{2}$ induced oxidative stress and apoptosis through upregulation of HO-1 expression via activation of the PI3K/ Akt / Nrf 2 pathway," Phytotherapy Research, vol. 27, no. 12, pp. 1810-1818, 2013.

[17] W. Su, A. Sun, D. Xu et al., "Tongxinluo inhibits oxidized lowdensity lipoprotein-induced maturation of human dendritic cells via activating peroxisome proliferator-activated receptor gamma pathway," Journal of Cardiovascular Pharmacology, vol. 56, no. 2, pp. 177-183, 2010.

[18] A. Sun, H. Liu, S. Wang et al., "Salvianolic acid B suppresses maturation of human monocyte-derived dendritic cells by activating PPAR $\gamma$," British Journal of Pharmacology, vol. 164, no. 8, pp. 2042-2053, 2011.

[19] I. G. Habeos, P. G. Ziros, D. Chartoumpekis, A. Psyrogiannis, V. Kyriazopoulou, and A. G. Papavassiliou, "Simvastatin activates Keap 1/Nrf2 signaling in rat liver," Journal of Molecular Medicine, vol. 86, pp. 1279-1285, 2008.

[20] D. Chartoumpekis, P. G. Ziros, A. Psyrogiannis, V. Kyriazopoulou, A. G. Papavassiliou, and I. G. Habeos, "Simvastatin lowers reactive oxygen species level by Nrf2 activation via PI3K/Akt pathway," Biochemical and Biophysical Research Communications, vol. 396, no. 2, pp. 463-466, 2010.

[21] A. Yilmaz, C. Reiss, A. Weng et al., "Differential effects of statins on relevant functions of human monocyte-derived dendritic cells," Journal of Leukocyte Biology, vol. 79, pp. 529-538, 2006.

[22] M. Lechmann, N. Shuman, A. Wakeham, and T. W. Mak, "The CD83 reporter mouse elucidates the activity of the CD83 promoter in B, T, and dendritic cell populations in vivo," Proceedings of the National Academy of Sciences of the United States of America, vol. 105, no. 33, pp. 11887-11892, 2008.

[23] R. Elgueta, M. J. Benson, V. C. de Vries, A. Wasiuk, Y. Guo, and R. J. Noelle, "Molecular mechanism and function of 
CD40/CD40L engagement in the immune system," Immunological Reviews, vol. 229, no. 1, pp. 152-172, 2009.

[24] V. Verhasselt, C. Buelens, F. Willems, D. de Groote, N. HaeffnerCavaillon, and M. Goldman, "Bacterial lipopolysaccharide stimulates the production of cytokines and the expression of costimulatory molecules by human peripheral blood dendritic cells: evidence for a soluble CD14-dependent pathway," Journal of Immunology, vol. 158, no. 6, pp. 2919-2925, 1997.

[25] J. Zhang and S. D. Crowley, "The role of type 1 angiotensin receptors on $\mathrm{T}$ lymphocytes in cardiovascular and renal diseases," Current Hypertension Reports, vol. 15, no. 1, pp. 39-46, 2013.

[26] A. L. Eggler, K. A. Gay, and A. D. Mesecar, "Molecular mechanisms of natural products in chemoprevention: induction of cytoprotective enzymes by Nrf2," Molecular Nutrition and Food Research, vol. 52, no. 1, pp. S84-S94, 2008.

[27] C. Wang, P. Liu, and J. K. Liao, "Pleiotropic effects of statin therapy: molecular mechanisms and clinical results," Trends in Molecular Medicine, vol. 14, no. 1, pp. 37-44, 2008.

[28] S. Wassmann, U. Laufs, K. Müller et al., "Cellular antioxidant effects of atorvastatin in vitro and in vivo," Arteriosclerosis, Thrombosis, and Vascular Biology, vol. 22, no. 2, pp. 300-305, 2002.

[29] A. Virdis, M. F. Neves, F. Amiri, R. M. Touyz, and E. L. Schiffrin, "Role of $\mathrm{NAD}(\mathrm{P}) \mathrm{H}$ oxidase on vascular alterations in angiotensin II-infused mice," Journal of Hypertension, vol. 22, no. 3, pp. 535-542, 2004.

[30] M. Zhou, E. A. Jaimes, and L. Raij, "Vascular but not cardiac remodeling is associated with superoxide production in angiotensin II hypertension," Journal of Hypertension, vol. 23, no. 9, pp. 1737-1743, 2005.

[31] C. de Ciuceis, F. Amiri, P. Brassard, D. H. Endemann, R. M. Touyz, and E. L. Schiffrin, "Reduced vascular remodeling, endothelial dysfunction, and oxidative stress in resistance arteries of angiotensin II-infused macrophage colony-stimulating factor-deficient mice: evidence for a role in inflammation in angiotensin-induced vascular injury," Arteriosclerosis, Thrombosis, and Vascular Biology, vol. 25, no. 10, pp. 2106-2113, 2005.

[32] A. M. Briones, N. Rodríguez-Criado, R. Hernanz et al., "Atorvastatin prevents angiotensin II-induced vascular remodeling and oxidative stress," Hypertension, vol. 54, pp. 142-149, 2009.

[33] J. S. Lee and Y. J. Surh, "Nrf2 as a novel molecular target for chemoprevention," Cancer Letters, vol. 224, no. 2, pp. 171-184, 2005.

[34] S. X. Liu, Y. Zhang, Y. F. Wang et al., "Upregulation of heme oxygenase-1 expression by hydroxysafflor yellow A conferring protection from anoxia/reoxygenation-induced apoptosis in H9c2 cardiomyocytes," International Journal of Cardiology, vol. 160, no. 2, pp. 95-101, 2012.

[35] X. L. Chen, G. Dodd, S. Thomas et al., "Activation of Nrf 2/ARE pathway protects endothelial cells from oxidant injury and inhibits inflammatory gene expression," American Journal of Physiology. Heart and Circulatory Physiology, vol. 290, no. 5, pp. H1862-H1870, 2006.

[36] X. L. Chen and C. Kunsch, "Induction of cytoprotective genes through Nrf2/antioxidant response element pathway: a new therapeutic approach for the treatment of inflammatory diseases," Current Pharmaceutical Design, vol. 10, no. 8, pp. 879891, 2004.
[37] K. Ishikawa and Y. Maruyama, "Heme oxygenase as an intrinsic defense system in vascular wall: implication against atherogenesis." Journal of atherosclerosis and thrombosis, vol. 8, no. 3, pp. 63-70, 2001.

[38] K. Ishikawa, M. Navab, N. Leitinger, A. M. Fogelman, and A. J. Lusis, "Induction of heme oxygenase-1 inhibits the monocyte transmigration induced by mildly oxidized LDL," Journal of Clinical Investigation, vol. 100, no. 5, pp. 1209-1216, 1997. 


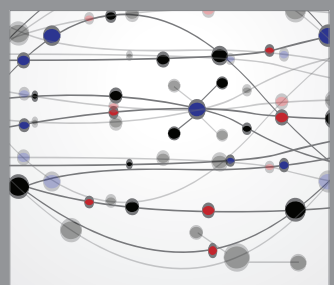

The Scientific World Journal
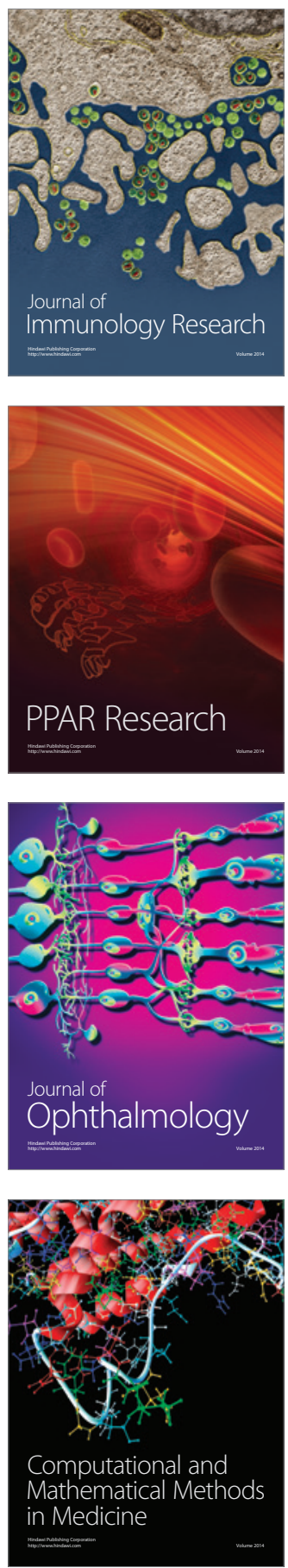

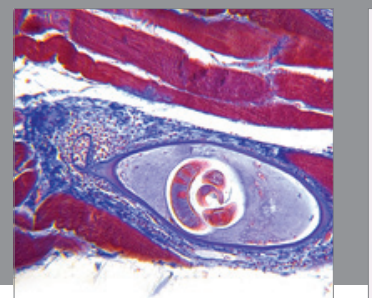

Gastroenterology

Research and Practice
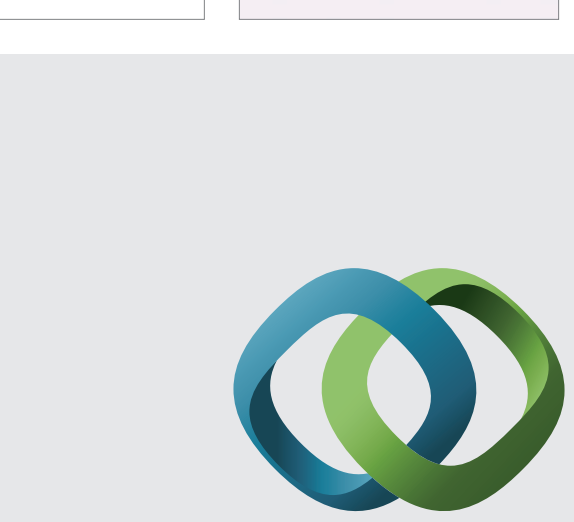

\section{Hindawi}

Submit your manuscripts at

http://www.hindawi.com
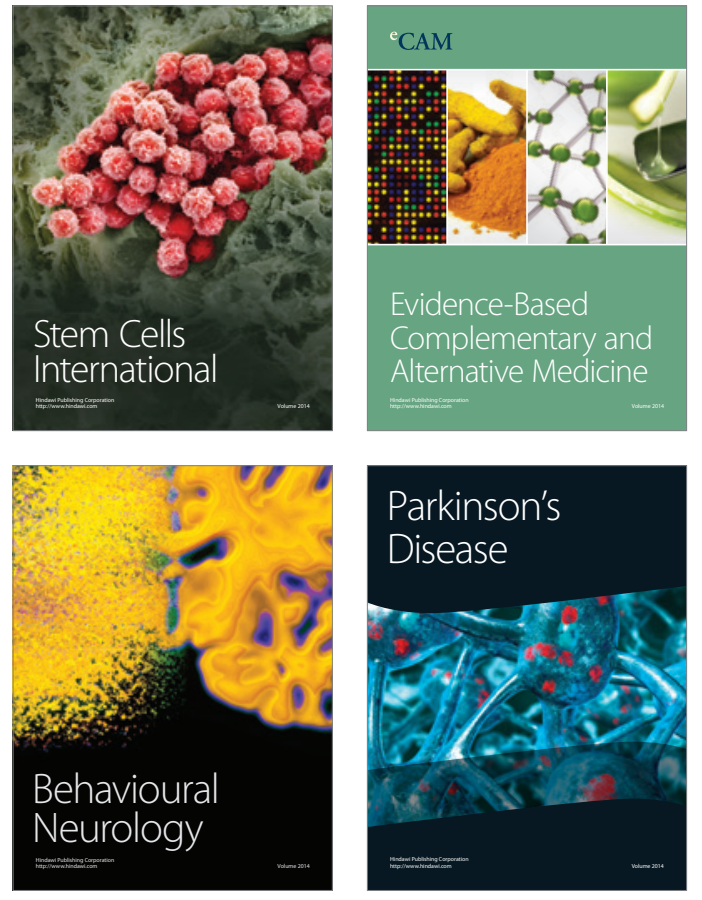
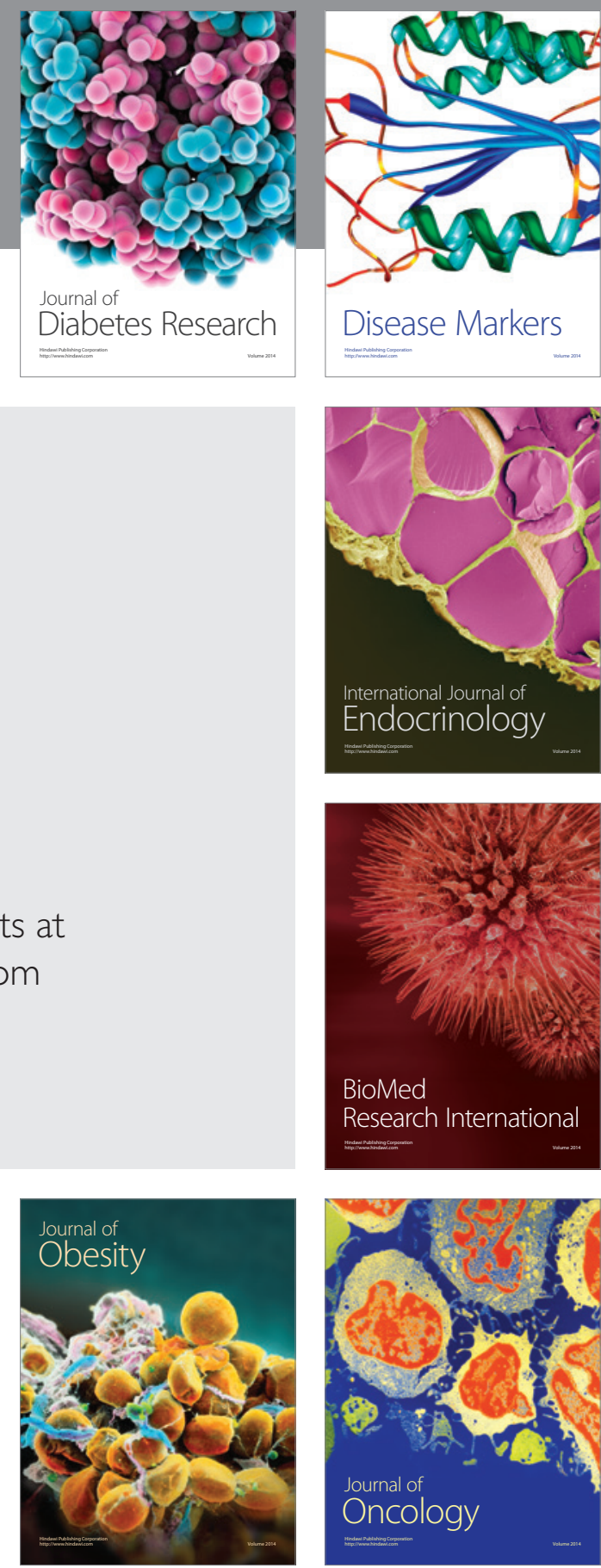

Disease Markers
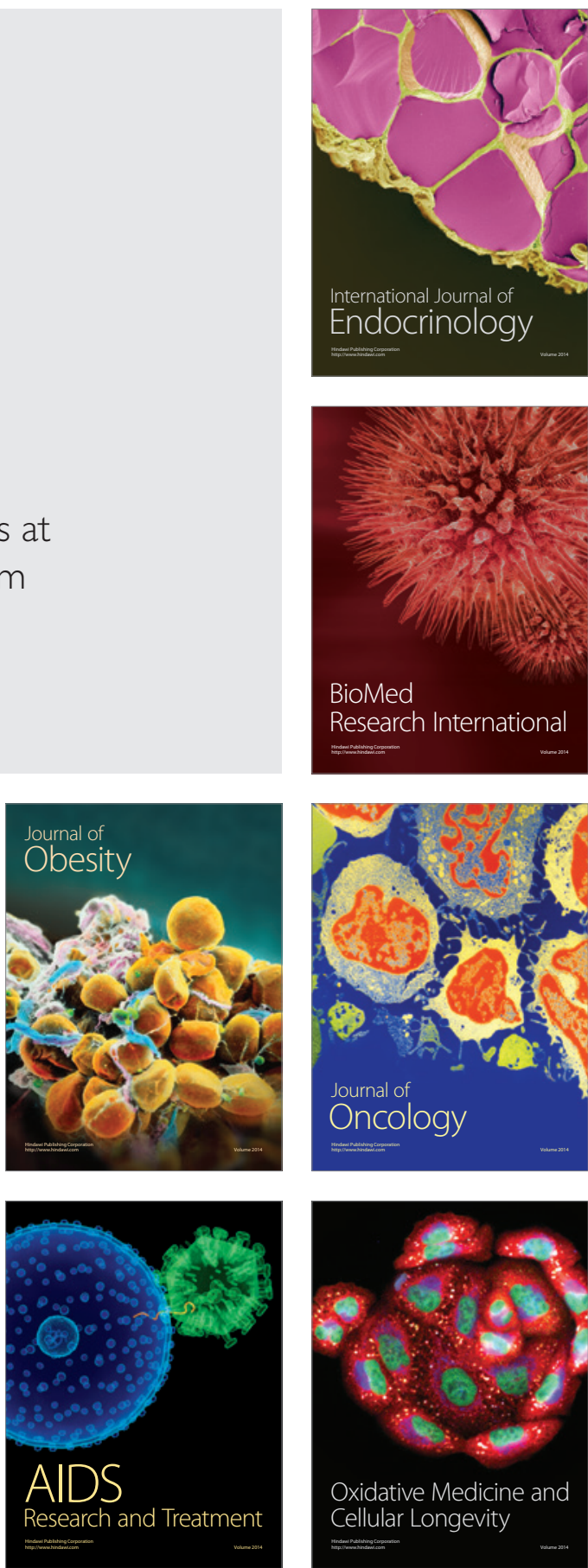